\title{
Characterization of Proteasome Inhibition on Astrocytes Cell Cycle
}

\author{
Qing-Guo Ren • Ying Yu • Xiang Luo $•$ Xie-Min Jie • \\ Deng-Ji Pan $\cdot$ Wei Wang
}

Published online: 13 February 2009

(C) Humana Press 2009

\section{Erratum to: J Mol Neurosci}

\section{DOI 10.1007/s12031-008-9161-8}

On the opening page of the original article, the name of Xiang Luo was omitted from the notice of equal contribution. Where is written "Qing-Guo Ren and Ying $\mathrm{Yu}$ contributed equally to this work" should be read "QingGuo Ren, Ying Yu and Xiang Luo contributed equally to this work." The Publisher regrets the error.

The online version of the original article can be found at http://dx.doi. org/10.1007/s12031-008-9161-8.

Qing-Guo Ren, Ying Yu and Xiang Luo contributed equally to this work.

Q.-G. Ren $\cdot$ Y. Yu $\cdot$ X. Luo $\cdot$ X.-M. Jie $\cdot$ D.-J. Pan $(\varangle) \cdot$

W. Wang $(\bowtie)$

Department of Neurology, Tongji Hospital, Tongji Medical,

College, Huazhong University of Science and Technology,

Wuhan 430030, People's Republic of China

e-mail: dengjipan@medmail.com.cn

e-mail:wwang_tjh@yahoo.com.cn

Q.-G. Ren

Department of Neurology, Hongqi Hospital, Mudanjiang Medical University,

Mudanjiang 157011, People's Republic of China 